\title{
Spontaneous dissection of native coronary arteries
}

\author{
R Butler, M W I Webster, G Davies, A Kerr, N Bass, G Armstrong, J T Stewart, P Ruygrok, \\ J Ormiston
}

Heart 2005;91:223-224

S pontaneous coronary artery dissection (SCAD) is relatively rare but occurs most often secondary to catheter tip or balloon injury. SCAD may affect women in the peripartum or early postpartum period. ${ }^{1}$ Women may dissect at a younger age ${ }^{2}$ and may dissect the left coronary artery more commonly than men.

\section{CASES}

We present 13 consecutive cases presenting in Auckland, New Zealand from 1996-2001. The diagnosis was made by angiography in 12 of the patients and at necropsy in one patient. Eleven of the 13 patients were female with a mean age of 44 years (range 25-58 years). There was no excess of traditional risk factors.

On presentation four of the 13 patients were not thought to have an ischaemic pain, but all subsequently had raised cardiac markers and/or ECG changes. One patient presented in the peripartum period and three patients had onset of chest pain after moderate to strenuous exercise.

Angiography demonstrated 22 dissected vessels in total (range 1-3); the vessels involved were the left main stem in two cases, left anterior descending (LAD) in 12 cases, intermediate in one case, circumflex in four cases and right coronary artery in four cases. The mean (SD) length of dissection was 43 (23) mm, which equates to 54 (30\%) of the total length of each artery. TIMI (thrombolysis in myocardial infarction) flow rates in the dissected vessels were TIMI 0-1 in four vessels, TIMI 2 in 10 vessels, and TIMI 3 in eight vessels.

Six subjects demonstrated a classical dissection flap (fig 1), nine subjects had a change in vessel calibre, abrupt in seven subjects and tapering in two subjects. Three subjects had both a typical flap and a change in vessel calibre. Both subjects with a tapering change in vessel calibre had contrasting hold up in the narrowed segment.

Two of three patients were successfully treated percutaneously with stent deployment. Three subjects underwent coronary artery bypass grafting, two successfully. Initial pharmacological treatment consisted of heparin in 10 patients, tirofiban in one patient, and thrombolysis in four patients. Discharge medication included aspirin in 11 patients and $\beta$ blockers in 11 patients.

The 11 subjects who survived to hospital discharge were followed up for an average of 13 months (range 2-36 months). All remain alive. Two developed further chest pain, one of whom has had a severe stenosis within the dissected vessel which had been successfully stented on a previous occasion

\section{DISCUSSION}

SCAD is a rare condition characterised by a dissection plane which usually runs in the outer media or between the media and adventitia. ${ }^{3}$ Haemodynamic compromise occurs as blood tracks in the dissection flap compressing the lumen.

The clinical features are typically of an acute coronary syndrome in a patient whose age and sex suggest a low

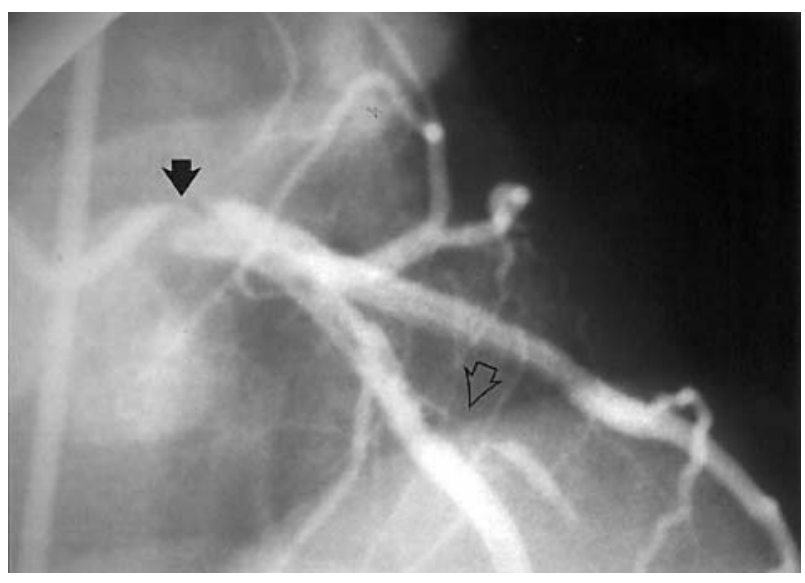

Figure 1 Spontaneous dissection involving the left main coronary artery (solid arrow) in case 6. The dissection extends down the LAD and circumflex arteries, occluding a diagonal vessel (open arrow).

probability of atherosclerotic disease. None of our patients described antecedent exertional angina.

Six of our patients presented with multiple dissections, suggesting a persistent vessel abnormality and a predisposition to dissection. Although there were differences in presentation, all had ischaemic episodes of recurrent unstable angina/infarction or sudden death. The natural history, particularly with proximal or extensive dissection, is unpredictable and warrants aggressive revascularisation.

Peripartum women may be at increased risk on two accounts: changes in blood flow increasing shear stress, and progesterone driven vessel wall changes which may predispose to coronary dissection. ${ }^{4}$ The suggestion of increased risk in this group was not seen in our series.

However, as dissections also occur in men and nonpregnant women, other factors must also be involved. The integrity of the vessel wall is important and inherited abnormalities such as Ehler-Dahnlos type IV or Marfan's syndrome increase the chance of dissection in any blood vessel including the coronary arteries. Pathological findings of cystic medial necrosis, typical of Marfan's syndrome, and an adventitial eosinophilic infiltrate are also associated with coronary dissection.

Other reported risk factors are strenuous exercise and haemodynamic stress which may be pharmacologically mediated. In our series, two patients developed symptoms during vigorous exercise at the gymnasium, and another soon after playing an international rugby match. Others document a spontaneous dissection in a cocaine addict.

Medical treatment includes antiplatelet treatment with aspirin. This appears logical as platelet-rich thrombi may develop secondary to transient or sustained vessel occlusion from the dissection flap. There are few data on the use of anticoagulants or glycoprotein IIb/IIIa inhibitors. Thrombolytic agents may restore vessel flow by lysing 
occlusive thrombi, with some case reports of therapeutic success. However, there are concerns that they may worsen the dissection process in a manner analogous to their deleterious effects in aortic dissection. Their use should be avoided when cardiac catheterisation facilities are available; in any case early catheterisation should be considered whenever the diagnosis is in doubt in young patients with a raised troponin value. $\beta$ Blockers are also used for their effects on heart rate, coronary blood flow, and vessel wall shear stress.

Percutaneous intervention has been increasingly performed for SCAD. The deployment of a stent has the advantage that the stent tacks back the dissection flap and prevents further dissection at that site. However, the stent can extrude intramural thrombus either up or downstream of the stent causing propagation of the dissection. The length of the dissection flap may also be difficult to judge angiographically and the potential for stent deployment into the false lumen exists, which can obliterate coronary flow. This can be minimised by injection of contrast through an infusion catheter or an over the wire balloon into the distal vessel before stent deployment. Unfortunately, free movement of the guidewire tip is not in itself sufficient proof that it is in the true lumen. The deployment of very long stents may be necessary when the dissection flap is extensive, although the use of drug eluting stents may reduce this concern.

Coronary artery bypass grafting has been used effectively and remains the treatment of choice for very extensive, multi-vessel dissection, particularly affecting the left main stem.

In conclusion, SCAD is a rare condition usually presenting as an acute coronary syndrome and should be considered in younger patients, especially those who develop chest pain in pregnancy or in the postpartum period. The condition is under-diagnosed and the natural history is unpredictable. Percutaneous coronary intervention is the treatment of choice, but needs careful attention to the position of the guidewire in the true lumen. Revascularisation does not alter the underlying propensity to further dissection present in some subjects.

\section{Authors' affiliations}

R Butler, Department of Cardiology, University Hospital of North Staffordshire, Stoke on Trent, UK

M W I Webster, N Bass, J T Stewart, P Ruygrok, J Ormiston, Auckland Hospital, Auckland, New Zealand

G Davies, Aintree NHS Trust, Liverpool, UK

A Kerr, Middlemore Hospital, Auckland, New Zealand

G Armstrong, North Shore Hospital, Auckland, New Zealand

Correspondence to: Dr Robert Butler, Department of Cardiology, University Hospital of North Staffordshire, Newcastle Road, Stoke on Trent, ST4 6QG; rob.butler@uhns.nhs.uk

Accepted 29 September 2003

\section{REFERENCES}

1 Tsimikas S, Giodano F, Tarazi R, et al. Spontaneous coronary artery dissection in patients with renal transplantation. J Invas Cardiol 1999; 11:316-21

2 DeMaio S, Kinsella S, Silverman M. Clinical course and long term prognosis of spontaneous coronary artery dissection. Am Heart J 1989;64:471-4.

3 Parry R, MacConnell T, Wilde P. Case report: spontaneous coronary artery dissection. Clin Radiol 1994;49:142-3.

4 Movsesian MA, Wray RB. Postpartum myocardial infarction. Br Heart J 1989;62:154-6.

\section{IMAGES IN CARDIOLOGY}

\section{Pseudoaneurysm compressing the ascending aorta on a recurrent aortic type A dissection}

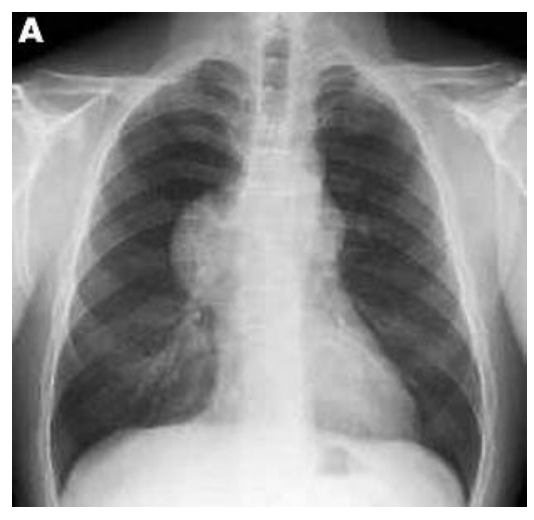

A

41 year old man was admitted for chest pain. A round shaped right hilar mass was seen on the chest $x$ ray (panel A). Seven years previously, he was operated on for a dissection of the ascending aorta with aortic insufficiency. A Medtronic valve graft No. 23 was used to replace the ascending aorta, while the transverse arch was not dissected at the time. Last year, he suffered an episode of sudden chest pain, but did not consult his physician.

ECG gated multislice computed tomography (MSCT) was performed with a volume zoom 4-slice scanner (Siemens, Erlangen, Germany). Full coverage of the thoracic aorta was obtained in a 47 second breath hold, with a $1.25 \mathrm{~mm}$ nominal slice thickness. MSCT angiography displayed a dissection of the aortic arch, and a posterior disruption of the distal suture of the prosthetic graft associated with a large impressive bilobular pseudoaneurysm compressing the ascending aorta (panel B). The patient was operated upon and a prosthetic replacement of the aortic arch was performed with reimplantation of the cerebral vessels.

Retrospectively, the chest pain was interpreted as a recurrence of the dissection in the aortic arch leading to a disruption of the distal suture and subsequently to the bilobular pseudoaneurysm.
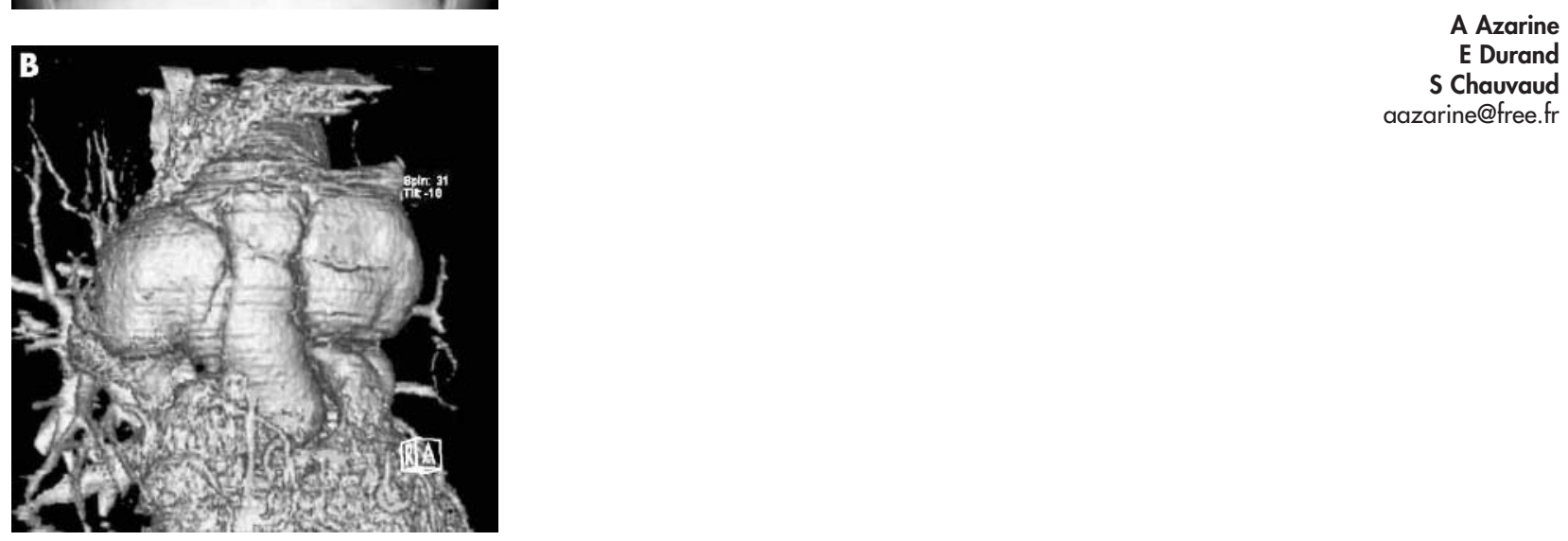